\title{
EVN observations of the Binary Black-Hole candidate SDSS J1536+0441
}

\author{
M. Bondi* \\ Istituto di Radioastronomia - INAF, 40129 Bologna, Italy \\ E-mail: bondieira.inaf.it
}

\section{Miguel A. Pérez-Torres}

Instituto de Astrofísica de Andalucía - CSIC, 18080 Granada, Spain

E-mail: torres@iaa.es

\begin{abstract}
We present European VLBI Network (EVN) observations at $5 \mathrm{GHz}$ of the candidate binary blackhole system SDSS J1536+0441. Both components, J1536+0441A and J1536+0441B, observed by the VLA at $8.5 \mathrm{GHz}$ and separated by 0.97 arcsec are detected with high $\mathrm{S} / \mathrm{N}$, proving the presence of two compact AGNs with radio luminosity $L_{R} \sim 10^{40} \mathrm{erg} \mathrm{s}^{-1}$. From a comparison with published $8.5 \& 22.5 \mathrm{GHz}$ flux densities, we derive an estimate of the radio spectral index of the two radio sources. Both sources have flat or inverted radio spectrum. In particular, J1536+0441A has a rising spectrum up to $\simeq 30 \mathrm{GHz}$, rest frame. Given the moderate brightness temperature derived from the flux and fitted size of J1536+0441A, we suggest that thermal free-free emission from an X-ray-heated disc may be powering the radio emission in J1536+0441A.
\end{abstract}

10th European VLBI Network Symposium and EVN Users Meeting: VLBI and the new generation of radio arrays

September 20-24, 2010

Manchester Uk

${ }^{*}$ Speaker. 


\section{Introduction}

SDSS J153636.22+044127.0 (hereafter SDSS J1536+0441) is one of the binary black-hole candidates found in a search for quasars displaying broad-lines features at different redshifts in the Sloan Digital Sky Survey [4]. The optical spectrum shows two sets of broad emission lines at $z=0.3889$ and $z=3727$, and a third set of narrow absorption lines at the intermediate redshift $z=0.3878$. Narrow emission lines were detected associated only with the higher redshift system. Radio observations carried out by the VLA at $8.5 \mathrm{GHz}$ detected two components, separated by $0^{\prime \prime} .97$ (5.1 kpc), named J1536+0441A and J1536+0441B respectively [14]. High resolution optical imaging detected the optical counterpart of J1536+0441B, but any optical signature of an AGN in this object was unclear and not conclusive [7,11]. Different interpretations were suggested in order to explain the rather complex optical features.

1. A black-hole binary (BHB) system within the same galaxy with a separation of $0.1 \mathrm{pc}$ and masses of $\sim 10^{7}$ and $\sim 10^{9} \mathrm{M}_{\odot}$ each with its own broad-line region and sharing the same narrow-line region $[4,11]$. In this scenario J1536+0441B is an elliptical galaxy, that can be responsible for the absorption features detected in the overall optical spectrum but is not contributing to the broad-line emission.

2. A AGN pair separated by $5.1 \mathrm{kpc}$ and probably residing in a moderately rich cluster of galaxies [7].

3. A double peaked emitter, albeit a peculiar one $[5,6,9]$.

All these scenarios are critically discussed by Lauer \& Boroson [11].

\section{EVN Observations}

To discriminate between the different interpretations given in the previous section is beyond the capability of the VLBI. An angular resolution of 0.02 mas coupled with a sensitivity of a few tens of microJy or better would be necessary to resolve and image the $0.1 \mathrm{pc} \mathrm{BHB}$, and this is not yet possible. Nonetheless, pc-scale imaging of J1536+0441 can shed some light on the nature of the two radio sources. We decided to target SDSS J1536+0441 exploiting the high sensitivity of the EVN with the following main goals:

- Pinpoint the AGN/AGNs position.

- Identify the nature of the radio emission associated to the component J1536+0441B. At the time of our EVN proposal no optical counterpart was known for J1536+0441B.

- Image the parsec scale structure of J1536+0441A and J1536+0441B and derive the radio spectral index properties using the available VLA/VLBA data.

To fulfill these goals we observed SDSS J1536+0441 at $5 \mathrm{GHz}$ on 2009 October 23 for about 5 hours (on-source time). The observations were carried out at the $1024 \mathrm{Mbit} \mathrm{s}^{-1}$ sustained bit rate reaching a $1 \sigma$ r.m.s sensitivity of about $15 \mu \mathrm{Jy} / \mathrm{beam}$ with a resolution of about 10 mas. More details on the observations, data analysis and discussion can be found in [3]. 


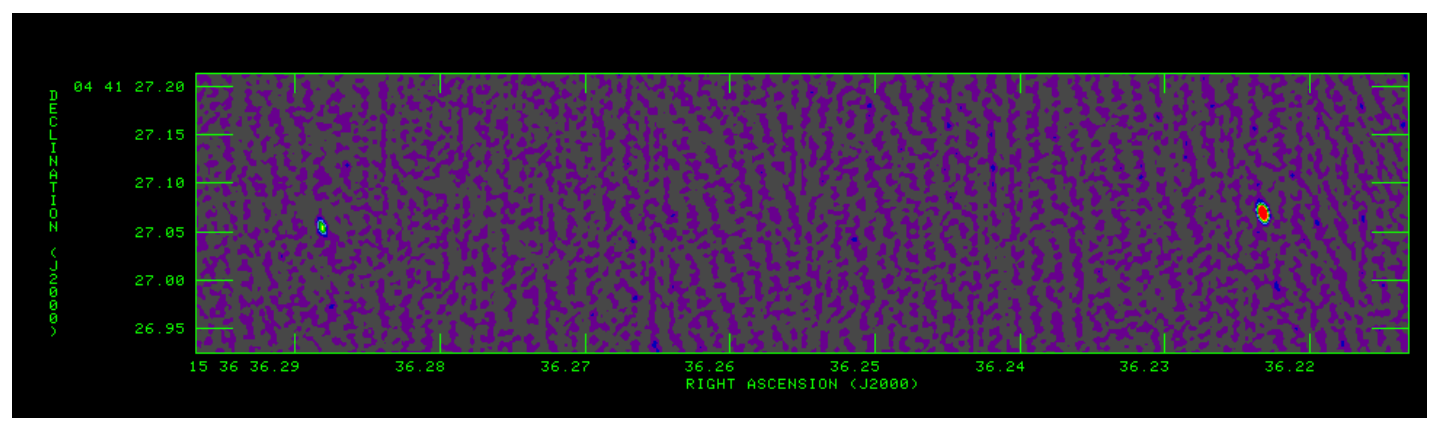

Figure 1: EVN image at $5 \mathrm{GHz}$ of the SDSS J1536+0441 field. This image was obtained applying natural weighting yielding to a resolution of $14 \times 7$ mas at position angle $16^{\circ}$. The $1 \sigma$ r.m.s. noise is $15 \mu \mathrm{Jy} / \mathrm{beam}$. $\mathrm{J} 1536+0441 \mathrm{~A}$, on the right, has a peak flux of $0.62 \mathrm{mJy} / \mathrm{beam}, \mathrm{J} 1536+0441 \mathrm{~B}$, on the left, has a peak flux of $0.22 \mathrm{mJy} / \mathrm{beam}$.

\section{Results \& Discussion}

Figure 1 shows the $5 \mathrm{GHz}$ EVN image of the J1536+0441 field. We detected with high signal-to-noise ratio both J1536+0441A and J1536+0441B ( $\mathrm{S} / \mathrm{N}=40$ and $\mathrm{S} / \mathrm{N}=15$, respectively). The field was also imaged with a different weighting scheme allowing better resolution $(\sim 7 \times$ 5 mas) but a larger noise. The sources were fitted with two-dimensional elliptical Gaussians obtaining consistent results between images with different resolutions. The derived flux densities at $5 \mathrm{GHz}$, positions and errors are: for J1536+0441A $S=0.72 \pm 0.06 \mathrm{mJy}, \alpha(J 2000)=$ $15^{\mathrm{h}} 36^{\mathrm{m}} 36^{\mathrm{s}} .2232, \delta(J 2000)=+04^{\circ} 41^{\prime} 27^{\prime \prime} .069$, and $\sigma_{\mathrm{VLBI}}=0.003$ mas; for J1536+0441B, $S=$ $0.24 \pm 0.03 \mathrm{mJy}, \alpha(J 2000)=15^{\mathrm{h}} 36^{\mathrm{m}} 36^{\mathrm{s}} .2881, \delta(J 2000)=+04^{\circ} 41^{\prime} 27^{\prime \prime} .054$, and $\sigma_{\mathrm{VLBI}}=0.003$ mas. J1536+0441A appears slightly resolved with a deconvolved fitted size $\simeq 3.2 \times 2.5$ mas with an estimated error of 1 mas.

In Table 1 we have collected the radio flux densities available at different frequencies in the literature for J1536+0441A and J1536+0441B. Table 1 lists measurements made with different resolutions (arcsec scale for VLA obervations compared to milli-arsec scale for VLBI observations) and usually at different epochs. While it can be dangerous to draw conclusions on the details of the radio spectral properties of J1536+0441A and J1536+0441B, these data are adequate to describe the general trend of the spectral index of the two components. Moreover, the VLBA observations at $8.5 \mathrm{GHz}$ [15] have been carried out 9 days earlier than our $5 \mathrm{GHz}$ EVN observations and with a similar resolution (within a factor of a few), therefore these flux densities can be considered unaffected by variability or resolution issues.

J1536+0441 is not detected in the FIRST survey [13], and the flux density at $1.4 \mathrm{GHz}$ in Table 1 is a $4 \sigma$ upper limit at the position of the radio source. Since the resolution of the FIRST survey is 5 arcsec the upper limit includes both the radio components.

As far as concern J1536+0441B the only thing we can say is that the data so far available suggest a flat radio spectrum between $1.4 \mathrm{GHz}$ and $8.5 \mathrm{GHz}$. The optical counterpart of J1536+0441B is very red $[7,11]$ and the detection of a pc-scale unresolved flat spectrum radio core with observed radio luminosity $L_{R}=v L_{v}=0.6 \times 10^{40} \mathrm{erg} \mathrm{s}^{-1}$ at $5 \mathrm{GHz}$ is best interpreted as an obscured AGN rather than an elliptical galaxy.

The almost contemporaneous radio flux densities measured on similar scales at 5 and $8.5 \mathrm{GHz}$ 


\begin{tabular}{cccccc}
\hline Comp. & $\begin{array}{c}1.4 \mathrm{GHz} \text { VLA } \\
\mathrm{mJy}\end{array}$ & $\begin{array}{c}5 \mathrm{GHz} \text { VLBI } \\
\mathrm{mJy}\end{array}$ & $\begin{array}{c}8.5 \mathrm{GHz} \text { VLBI } \\
\mathrm{mJy}\end{array}$ & $\begin{array}{c}8.5 \mathrm{GHz} \text { VLA } \\
\mathrm{mJy}\end{array}$ & $\begin{array}{c}22.5 \mathrm{GHz} \text { VLA } \\
\mathrm{mJy}\end{array}$ \\
\hline J1536+0441A & $<0.6(\mathrm{~A}+\mathrm{B})$ & $0.72 \pm 0.06$ & $0.88 \pm 0.12$ & $1.17 \pm 0.04$ & $1.65 \pm 0.11$ \\
$\mathrm{~J} 1536+0441 \mathrm{~B}$ & $<0.6(\mathrm{~A}+\mathrm{B})$ & $0.24 \pm 0.03$ & & $0.27 \pm 0.02$ & \\
\hline
\end{tabular}

Table 1: Radio flux densities of J1536+0441A and J1536+0441B. The $1.4 \mathrm{GHz}$ flux density is an upper limit from the FIRST Survey [13] including both components. The $8.5 \mathrm{GHz}$ VLBI flux is from VLBA observations made in October 2009 [15]. The $8.5 \mathrm{GHz}$ VLA flux is from [14]. The $22.5 \mathrm{GHz}$ VLA flux is from [15].

allows us to better constrain the spectral shape of J1536+0441A. The radio spectrum is clearly inverted (with a spectral index $\alpha \simeq 0.4$ with $S(v) \propto v^{\alpha}$ ). The inverted spectrum is confirmed by the non-contemporaneous VLA data, with $\alpha=0.35 \pm 0.08$ between $8.5 \mathrm{GHz}$ and $22.5 \mathrm{GHz}$ [15], yielding to an inverted spectrum up to $\simeq 30 \mathrm{GHz}$ rest-frame.

One possible origin of the inverted radio spectrum is synchrotron self-absorption. For selfabsorption to occur, the brightness temperature must be comparable to the kinetic temperature of the synchrotron electrons. The measured brightness temperature for J1536+0441A, for which we have a deconvolved fitted size, is $T_{b}=9 \times 10^{6} \mathrm{~K}$. This value is too low to affect the radio spectrum unless the magnetic field is rather large [8]. An alternative interpretation of the inverted radio spectrum of J1536+0441A is thermal free-free emission from a disk wind [8, 2]. As shown in the model developed by Blundell \& Kuncic [2], high brightness temperatures $\left(T_{b} \sim 10^{7} \mathrm{~K}\right)$ can arise from a thermal plasma provided that it is hot $\left(T_{e} \gtrsim 10^{7} \mathrm{~K}\right)$ and marginally optically thin $\left(\tau_{v}^{f f} \lesssim 1\right)$. In this scenario, we can expect a link between the radio and $\mathrm{X}$-ray emission. A correlation between radio luminosity at $5 \mathrm{GHz}\left(L_{R}\right)$ and bolometric $0.2-20 \mathrm{keV} \mathrm{X}$-ray luminosity $\left(L_{X}\right)$, with $L_{R} / L_{X} \sim$ $10^{-5}$ indeed has been found in nearby Seyferts and radio quiet quasars [12, 10]. For J1536+0441 A the radio luminosity at $5 \mathrm{GHz}$, as derived from our observations is $L_{R}=v L_{5 \mathrm{GHz}}=1.9 \times 10^{40} \mathrm{erg}$ $\mathrm{s}^{-1}$. Using the X-ray luminosity measured by Swift [1], we obtain $L_{R} / L_{X}=1.4 \times 10^{-5}$, assuming all the X-ray emission is associated to J1536+0441A.

\section{Summary}

We have presented the $5 \mathrm{GHz}$ EVN observations of the candidate binary black-hole system J1536+0441. We have detected two compact cores associated with the sources J1536+0441A and J1536+0441B separated by $0.97 \operatorname{arcsec}(5.1 \mathrm{kpc})$. The main results can be summarized as follows.

1. A flat spectrum radio nucleus, unresolved on the pc-scale, and with a radio luminosity of $0.6 \times 10^{40} \mathrm{erg} \mathrm{s}^{-1}$ is found at the position of $\mathrm{J} 1536+0441 \mathrm{~B}$, suggesting that $\mathrm{J} 1536+0441 \mathrm{~B}$ is most likely an obscured AGN rather than a passive elliptical galaxy.

2. At the position of J1536+0441A we detect a slightly resolved radio nucleus. From almost contemporaneous $5 \& 8.5 \mathrm{GHz}$ observations we derive a spectral index of $\alpha \simeq 0.4$ (with $\left.S_{v} \propto v^{\alpha}\right)$. From a comparison with VLA observations the rising spectrum continues up to $22.5 \mathrm{GHZ}$ ( $\simeq 30 \mathrm{GHz}$ rest frame). Given the rather modest measured brightness temperature $\left(T_{b}=9 \times 10^{6} \mathrm{~K}\right)$, we suggest that the inverted spectrum can be explained not as synchrotron self-absorption but as thermal free-free emission from a disk wind. 
3. We derive a value of $L_{R} / L_{X}=1.4 \times 10^{-5}$ that is totally consistent with the correlation found for radio-quiet quasars and Seyfert galaxies.

Acknowledgments MB acknowledges support from the contract PRIN-INAF 2008. MAPT acknowledges support by the Spanish Ministry of Science and Innovation, and from the Andalusian Research Ministry, through grants AYA2009-13036-C02-01 and TIC-126. The European VLBI Network is a joint facility of European, Chinese, South African and other radio astronomy institutes funded by their national research councils. We wish to thank all the people at the telescopes involved in the observations, those who took care to correlate and process the data, and in particular Dr. Yurii Pidopryhora, support scientist for these observations.

\section{References}

[1] Z. Arzoumanian et al. 2009, Swift Observation of the Candidate Supermassive Black Hole Binary SDSS J153636.22+044127.0, ATel, 1931

[2] K. M. Blundell \& Z. Kuncic 2007, On the Origin of Radio Core Emission in Radio-quiet Quasars, ApJ, 668, L103

[3] M. Bondi \& M.-A. Pérez-Torres 2010, VLBI Detection of an Active Galactic Nucleus Pair in the Binary Black Hole Candidate SDSS J1536+0441, ApJ, 714, L271

[4] T.A. Boroson \& T.R. Lauer 2009, A candidate sub-parsec supermassive binary black hole system, Nature, 458,53

[5] R. Chornock et al.2009, SDSS J1536+0441: An Extreme "Double-peaked Emitter," Not a Binary Black Hole, ATel, 1955

[6] R. Chornock et al.2010, The Quasar SDSS J1536+0441: An Unusual Double-peaked Emitter, ApJ, 709,39

[7] R. Decarli et al. 2009, Probing the Nature of the Massive Black Hole Binary Candidate SDSS J1536+0441, ApJ, 703, L76

[8] J.F. Gallimore et al. 1996, The Subarcsecond Radio Structure in NGC 1068. II. Implications for the Central Engine and Unifying Schemes, ApJ, 464, 198

[9] C.M. Gaskell 2010, Close supermassive binary black holes, Nature, 463, 1

[10] A. Laor \& E. Behar 2008, On the origin of radio emission in radio-quiet quasars, MNRAS, 390, 847

[11] T.R. Lauer \& T.A. Boroson 2009, Hubble Space Telescope Images and KPNO Spectroscopy of the Binary Black Hole Candidate SDSS J153636.22+044127.0, ApJ, 703, 930

[12] F. Panessa et al. 2007, The X-ray and radio connection in low-luminosity active nuclei, A\&A, 467, 519

[13] R.L. White et al. 1997, A Catalog of 1.4 GHz Radio Sources from the FIRST Survey, ApJ, 475, 479

[14] J.M. Wrobel \& A. Laor 2009, Discovery of Radio Emission from the Quasar SDSS J1536+0441, a Candidate Binary Black Hole System, ApJ, 699, L22

[15] J.M. Wrobel \& A. Laor 2010, Parsec-scale Localization of the Quasar SDSS J1536+0441A, a Candidate Binary Black Hole System, ApJ, 714, L295 